\title{
Cell type-specific expression and localization of cytochrome P450 isoforms in tridimensional aggregating rat brain cell cultures
}

\author{
S. Vichi ${ }^{\mathrm{a}, *, 1}$, J. Sandström von Tobel ${ }^{\mathrm{b}, \mathrm{c}, 1}$, S. Gemma ${ }^{\mathrm{a}}$, S. Stanzel ${ }^{\mathrm{d}}$, A. Kopp-Schneider ${ }^{\mathrm{d}}$, \\ F. Monnet-Tschudi ${ }^{\mathrm{b}, \mathrm{c}}$, E. Testai ${ }^{\mathrm{a}, 1}$, M.G. Zurich ${ }^{\mathrm{b}, \mathrm{c}, 1}$ \\ a Istituto Superiore di Sanità, Environment and Primary Prevention Department, Mechanisms of Toxicity Unit Rome, Italy \\ ${ }^{\mathrm{b}}$ Department of Physiology, University of Lausanne, Lausanne, Switzerland \\ ' Swiss Center for Applied Human Toxicology (SCAHT), Switzerland \\ d Department of Biostatistics, German Cancer Research Center, Heidelberg, Germany
}

\section{A R T I C L E I N F O}

\section{Article history:}

Received 15 July 2014

Accepted 6 March 2015

Available online $\mathrm{xxxx}$

\section{Keywords:}

Cytochrome P450

3D aggregating brain cell cultures

Biokinetics

\begin{abstract}
A B S T R A C T
Within the Predict-IV FP7 project a strategy for measurement of in vitro biokinetics was developed, requiring the characterization of the cellular model used, especially regarding biotransformation, which frequently depends on cytochrome P450 (CYP) activity. The extrahepatic in situ CYP-mediated metabolism is especially relevant in target organ toxicity. In this study, the constitutive mRNA levels and protein localization of different CYP isoforms were investigated in 3D aggregating brain cell cultures. CYP1A1, CYP2B1/B2, CYP2D2/4, CYP2E1 and CYP3A were expressed; CYP1A1 and 2B1 represented almost 80\% of the total mRNA content. Double-immunolabeling revealed their presence in astrocytes, in neurons, and to a minor extent in oligodendrocytes, confirming the cell-specific localization of CYPs in the brain. These results together with the recently reported formation of an amiodarone metabolite following repeated exposure suggest that this cell culture system possesses some metabolic potential, most likely contributing to its high performance in neurotoxicological studies and support the use of this model in studying brain neurotoxicity involving mechanisms of toxication/detoxication.
\end{abstract}

(C) 2015 Published by Elsevier Ltd.

\section{Introduction}

The cytochrome P450 (CYP) superfamily is one of the most important groups of enzymes involved in the biotransformation of a large number of endogenous and exogenous compounds, including toxic substances, drugs and environmental chemicals. Although the liver is the primary organ responsible for CYP-mediated metabolism, the relevance of extrahepatic CYPmediated metabolism is widely recognized, especially regarding in situ metabolism in target organ toxicity (Pavek and Dvorak,

Abbreviations: CYP, cytochrome P450; 3D, three-dimensional; DIV, day in vitro; $\mathrm{Ct}$, threshold cycle; CV, coefficient of variation; PBS, phosphate buffered saline; GFAP, glial fibrillary acidic protein; MBP, myelin basic protein; MAP-2, MicrotubuleAssociated Protein 2; SD, standard deviation; LOEC, Lowest Observed Effect Concentration; NF, neurofilaments; CNS, central nervous system.

* Corresponding author at: Istituto Superiore di Sanità, Environment and Primary Prevention Department, Viale Regina Elena, 299, 00161 Rome, Italy. Tel.: +39 06 49903852.

E-mail address: susanna.vichi@iss.it (S. Vichi).

1 These authors equally contributed to the work.
2008; Ravindranath and Strobel, 2013; Rieder et al., 1998). Neurotoxicity is one of the most relevant toxicological end-points, due to the crucial role played by the central nervous system (CNS) in the organism functionality.

In the CNS CYPs have been identified as functional enzymes, and are known to metabolize in situ a variety of compounds including centrally acting drugs, xenobiotics, neurotoxins, endogenous steroids and neurochemicals (Ravindranath and Strobel, 2013; Zanger et al., 2004). Different CYP isoforms have been identified in the rat CNS using in situ hybridization, catalytic, molecular, and immunohistochemical techniques. Results showed that drugmetabolizing CYPs are not homogeneously distributed among brain regions, which differ widely in cellular composition, cell density and function. Indeed, they are characterized by a marked region-specific distribution, and have been found in neurons, glial cells and at the blood-brain interface (Meyer et al., 2007; Stamou et al., 2014).

The expression of CYPs in the whole brain is reported to occur at only $1 \%$ of the levels found in the liver, therefore it is unlikely that brain CYPs contribute to overall clearance of any xenobiotic. 
However, since CYPs can be highly expressed in specific cells or brain regions, the metabolism of drugs crossing the blood-brain barrier can be of relevance and responsible of specific effects locally within the brain (Miksys and Tyndale, 2002, 2004).

Similarly to the liver, brain CYPs are responsive to chemicals: induction can be regulated by several complex mechanisms (including the increase of mRNA expression) (Johri et al., 2007; Parmar et al., 2003) which are both tissue and cell-type specific (Miksys and Tyndale, 2004). Brain CYPs can be responsive to the same inducers active on hepatic CYPs (Ghersi-Egea et al., 1987; Kapoor et al., 2006; Joshi and Tyndale, 2006), although different induction patterns have been reported (Miksys et al., 2000; Stamou et al., 2014).

It has been stated that results from in vivo toxicity tests are not always optimal for neurotoxic effects (Harry and TiffanyCastiglioni, 2005), due to the inability to have factors as neuronal, hormonal and immunological stimuli under full experimental control. Indeed, some degree of over-prediction of neurotoxic effects was reported with 45 miscellaneous drugs, particularly when high doses were tested, showing that high-dose effects such as ataxia and convulsions in animals might not be relevant in humans (Fletcher, 1978). Despite the market for drugs used to cure CNS disorders is set to grow substantially in the coming years, due to an aging population, CNS drugs have a low chance of success in drug development. This is explained by the complexity of the brain and the liability of CNS drugs to cause CNS side effects, not always identified by traditional neurotoxicity testing for the above-mentioned reasons. These observations, together with an increasing demand for reduction of animal use in toxicological testing, strongly push toward the development of suitable in vitro methods for neurotoxicity testing, able to predict neurotoxicity in the early stage of drug development.

Among several in vitro models used to mimic the brain, aggregating brain cell cultures represent most closely the multicellular architecture, maturation, and functions of the in vivo brain tissue. These three-dimensional (3D) cell cultures have been shown to reach a highly differentiated phenotype that is maintained for at made them a precious and useful model system for neurotoxicological investigations (Honegger et al., 2011; Honegger and Werffeli, 1988; Zurich et al., 2000, 2013). Although the presence of CYP activity has previously been suggested (Monnet-Tschudi et al., 2008, 2000), scant information is available on the metabolic capability of these 3D cultures, as for most of the available in vitro models for neurotoxicity.

Generally speaking, the absence of characterization of the metabolic competence of the model used, as well as of information on the compounds permeability through the blood brain barrier, strongly limits the interpretation of results of in vitro neurotoxicity funded this study, had the overall aim of improving the assessment of drug safety, by integrating kinetics and dynamics data. Within the project a strategy for the measurement of in vitro biokinetics was developed which foresees the characterization of the different cellular models used.

In this study, we used a combination of quantitative Reverse Transcription-PCR (qRT-PCR), immunohistochemistry and western-blot techniques to characterize the expression and the presence of CYP isoforms and their cell type-specific localization in 3D aggregating brain cell cultures. In addition, we explored the responsiveness of the cells to nicotine to evaluate possible induction of a panel of different CYPs. Nicotine was selected as a model inducer, since it has been reported to induce several CYPs in some rat brain regions after chronic exposure in vivo (Ande et al., 2012; Joshi and Tyndale, 2006; Miksys et al., 2000; Yue et al., 2008). least two months (Zurich et al., 1998). These characteristics have for risk assessment purposes. The EU FP7 Project Predict-IV, which

\section{Materials and methods}

\subsection{Cell culture and treatments}

Serum-free, rotation-mediated aggregating brain cell cultures were prepared from 16-day embryonic rat brain (Janvier Labs, France), as described previously in detail (Honegger et al., 2011, 1979). The dissected brains from about 100 embryos - comprising the telencephalon, mesencephalon and rhombencephalon - were dissociated mechanically into a single cell fraction by the sequential passage through nylon sieves of $200-\mu \mathrm{m}$ and $100-\mu \mathrm{m}$ pore sizes. Through all steps of the preparation, the cells were kept in ice-cold, $\mathrm{Ca}^{2+}-\mathrm{Mg}^{2+}$-free saline (Puck's saline solution $\mathrm{D}_{1}$ ). The dissociated cells were washed by centrifugation ( $\left.15 \mathrm{~min}, 300 \mathrm{~g}_{\max }\right)$, and finally resuspended in cold serum-free culture medium (modified DMEM). Aliquots of the cell suspension ( $4 \mathrm{ml}$, on average containing the amount of cells from one embryonic brain) were distributed in 100 culture flasks (25-ml modified Erlenmeyer flasks with air-permeable stoppers) and incubated under continuous gyratory agitation $(68 \mathrm{rpm})$ in a $\mathrm{CO}_{2}$ incubator $\left(10 \% \mathrm{CO}_{2}, 90 \%\right.$ humidified air, $37^{\circ} \mathrm{C}$ ). After two days, the cultures were transferred to larger flasks (50-ml modified Erlenmeyer flasks with air-permeable stoppers) and supplemented with $4 \mathrm{ml}$ of fresh medium. The frequency of gyratory agitation was increased progressively from $68 \mathrm{rpm}$ at culture initiation day in vitro (DIV) 0 , to $70 \mathrm{rpm}$ (evening of DIV 0), then to $74 \mathrm{rpm}$ (DIV 1), $78 \mathrm{rpm}$ (DIV 2, after the culture transfer), and then to the final speed of $80 \mathrm{rpm}$ (DIV 4) which was kept throughout the following culture period. Culture media was replenished by the exchange of $5 \mathrm{ml}$ of medium (of a total of $8 \mathrm{ml}$ ) per flask, at intervals of 3 days until DIV 14, and at intervals of 2 days thereafter. For experimentation, the aggregates of several original flasks were pooled and redistributed in order to prepare replicate cultures each containing about 200 free-floating aggregates (equivalent to about $1 / 6$ of the original flask). The replicates were kept under standard culture conditions.

Nicotine hydrogen tartrate salt was purchased from Sigma and stock solutions were prepared in $\mathrm{H}_{2} \mathrm{O}$ and neutralized against phenol red with $\mathrm{NaOH}$. Cultures were exposed to nicotine (50-200 $\mu \mathrm{M}$ ) during $4 \mathrm{~h}, 24 \mathrm{~h}$, and $48 \mathrm{~h}$. Aliquots of the stock solutions were added directly to the culture supernatants. Control cultures received an equal volume of the solvent. The final concentration of DMSO in the culture medium was $0.05 \%(\mathrm{~V} / \mathrm{V})$. For each group of treatment, 3 replicate cultures, coming from were performed at DIV20, and three experiments at DIV33. These experiments were performed on aggregates prepared from four independent pools of embryonic brains.

\subsection{RNA extraction and $c D N A$ synthesis}

Total mRNA was extracted from aggregating brain cell cultures using the QIAshredder and total RNeasy kits from Qiagen, according to manufacturer's instructions and eluted in RNase-free water. The RNA concentration and purity was determined by measuring UV absorbance at 260 and $280 \mathrm{~nm}$ using the NanoDrop ND-1000 UV-Vis Spectrophotometer (Nanodrop Technologies). cDNA was synthesized from $1 \mu \mathrm{g}$ of total RNA in a total volume of $20 \mu \mathrm{l}$ using the Applied High Capacity cDNA Reverse Transcription kit (Life Technologies). The obtained transcripts were stored at $-20^{\circ} \mathrm{C}$ and analyzed by qRT-PCR methodology.

\subsection{Quantitative real-time $P C R$}

Quantitative analysis of CYP mRNA expression was performed by qRT-PCR, by subjecting the obtained cDNA to PCR amplification the same pool of embryonic brains, were used. Three experiments 
using 96-well optical reaction plates in a StepOne ${ }^{\mathrm{TM}}$ Real-Time PCR System (Life Technologies).

The levels of CYP1A1, CYP1A2, CYP2B1, CYP3A1 and CYP3A2 mRNAs were determined using TaqMan chemistry according to the method described by Meredith et al. (2003), with thermal conditions of an initial two-step holding stage at $50{ }^{\circ} \mathrm{C}$ for $2 \mathrm{~min}$ and subsequent denaturation at $95^{\circ} \mathrm{C}$ for $10 \mathrm{~min}$, followed by 40 cycling stages of denaturation at $95^{\circ} \mathrm{C}$ for $15 \mathrm{~s}$ and annealing/extension at $60^{\circ} \mathrm{C}$ for $1 \mathrm{~min}$. TaqMan probes were labeled on the 5 '-end with FAM as the fluorophore dye and on the 3 '-end with TAMRA as the quencher dye.

The levels of CYP2D2, CYP2E1 and CYP2D4/2D6 mRNAs were measured applying SYBR-green chemistry following the method of Mrozikiewicz et al. (2010) and Yoshida et al. (2002) with the same thermal profile as described above for the PCR amplification, with the addition of a final melting curve stage.

Quantitation of copies of mRNA of each tested CYP was calculated from the experimental threshold cycle value $(\mathrm{Ct})$, by interpolation from the standard curve generated using serial dilution of known cDNA concentrations. For each tested CYP a five-point standard curve was constructed. The mRNA levels obtained for each CYP were normalized with respect to the mRNA levels determined for Cyclophilin A, selected as housekeeping gene. Primers and TaqMan probes for Cyclophilin A were designed using Primer Express v. 3.0 software (AB-Life Technologies) and custom-synthesized by AB-Life Technologies. Cyclophilin A was co-amplified in a simultaneous reaction together with the CYPs, under the same experimental conditions and with a similar PCR efficiency with respect to the target gene. Technical duplicates were performed for each real time reactions. After treatment with inducers, results of normalized mRNA expression levels were reported as fold change values with respect to untreated cultures. All primers and TaqMan probes used in this study are listed in Table 1. For each experiment, the coefficient of variation (CV) in the technical replicates $(n=3)$ on each replicate cultures was around $2 \%$.

\subsection{Immunohistochemistry}

Mature aggregates were washed twice with pre-warmed phosphate buffered saline (PBS), embedded in cryomatrix (Thermo Fisher Scientific), frozen in liquid nitrogen cooled isopentane, and stored at $-80^{\circ} \mathrm{C}$. Cryosections $(10 \mu \mathrm{m})$ were fixed for $10 \mathrm{~min}$ in $4 \%$ paraformaldehyde/PBS. Sections were blocked with normal horse-, goat- or rabbit serum (1:25 in PBS with $0.1 \%$ Triton-X100, Jackson), and subsequently incubated overnight at $4{ }^{\circ} \mathrm{C}$ with antibodies against CYP1A1 (H-70) (1:50, rabbit polyclonal, Santa Cruz), CYP2D4 (1:1000, rabbit polyclonal, Millipore), CYP2E1 (1:2000, rabbit polyclonal, Millipore), CYP2B1/2B2 (1:50, mouse monoclonal, Santa Cruz) and CYP3A (L-14) (1:200, goat polyclonal, Santa Cruz). Following, sections were incubated with the corresponding biotinylated IgG, i.e. horse anti-mouse IgG (1:200, Vector), goat anti-rabbit $\operatorname{IgG}(1: 200$ Vector) and rabbit anti-goat (1:200, Vector), followed by revelation with FITC-avidin (1:100, Vector). Determination of the cellular localization of CYP1A1, CYP2D4, CYP2E1 and CYP3A was achieved by double labeling with antibodies against astrocytic Glial Fibrillary Acidic Protein (GFAP) (1:800, mouse monoclonal, Sigma-Aldrich), neuronal phosphorylated and non-phosphorylated neurofilaments (1:1000, mouse monoclonal SMI31, Covance, and mouse monoclonal, Enzo Life Sciences) and oligodendrocytic myelin basic protein (MBP) (1:40, mouse monoclonal, Millipore). For CYP2B1/2B2, double labeling was done with anti GFAP $(1: 100$, rabbit polyclonal, Sigma-Aldrich) and Microtubule-Associated Protein 2 (MAP-2) (1:200, rabbit polyclonal, Chemicon). Alexa Fluor 546 donkey anti-mouse, respectively anti-rabbit, $\operatorname{IgG}(1: 400$, Life
Technologies) was used to reveal cell type-specific labeling. Hoechst (33342, Molecular Probes) was used to visualize cell nuclei. Sections were mounted in ProLong Gold antifade reagent (Life Technologies). Images were acquired on a Nikon Eclipse 90i fluorescence microscope. Automatic pixel density recognition of Hoechst labeled area, using Image software, was used to create a mask for visualization of nuclei (indicated by circular-like white lines in insets of Fig. 2).

\subsection{Western blotting and relative protein content measurement}

Mature aggregates were homogenized with glass-Teflon douncer in $50 \mathrm{mM} \mathrm{NaCl}, 20 \mathrm{mM}$ Tris- $\mathrm{HCl} \mathrm{pH} 7.4,1 \mathrm{mM}$ EDTA, $0.5 \%$ Sodium Deoxycholate 20 , and $1 \times$ complete protease inhibitor cocktail (Roche, Switzerland). Protein concentration was determined by absorbance at $280 \mathrm{~nm}$ using NanoDrop ND-1000 UV-Vis Spectrophotometer (Nanodrop Technologies). Lysates were diluted to the same total protein concentration, and a total quantity of $50 \mu \mathrm{g} / \mathrm{sample}$ and lane was loaded, mixed 1:6 with Laemmli buffer (375 mM Tris-HCl pH 6.8, 9\% SDS, 50\% Glycerol, 9\% Betamercaptoethanol, 0.03\% Bromophenol blue). Samples were separated on 4-15\% TX Page gel (BioRad). Proteins were transferred to $0.22 \mu \mathrm{m}$ pore size nitrocellulose membranes (BioRad, Switzerland) and subsequently stained with ponceau red to ensure equal loading and proper transfer. Membranes were washed and blocked over-night at $4{ }^{\circ} \mathrm{C}$ with $10 \%$ non-fat dry milk/20 in mM Trizma base, $137 \mathrm{mM} \mathrm{NaCl}, 0.05 \%$ Tween, $\mathrm{pH}$ 7.6. Membranes were probed ( $3 \mathrm{~h}$ at room temperature) with primary antibodies against CYP1A1 (H-70) (1:1000, sc-20772, rabbit polyclonal, Santa Cruz), CYP2D4 (1:1000, AB1272, rabbit polyclonal, Millipore), CYP2E1 (1:1000, AB1252, rabbit polyclonal, Millipore), CYP2B1/ 2B2 (9.14) (1:500, sc-73546, mouse monoclonal, Santa Cruz) and CYP3A1 (P6) (1:500, sc-53246, mouse monoclonal, Santa Cruz). Horseradish peroxidase conjugated secondary goat anti-rabbit, Advansta (1:10000) and goat anti-mouse, Bio-Rad (1:10000), antibodies were applied, and subsequently visualized with chemiluminescence (Western Bright ECL, Advansta). Autoradiograms were processed using ImageJ to determine densitometry units. For relative quantifications CYP-band intensity was normalized to its corresponding load on ponceau red, and thereafter fold changes were calculated to relative CYP-band intensities in liver. Three replicates were performed for liver, brain, and brain aggregates. For the semi-quantitative determination of CYP protein obtained by western blot, the relative band intensity values of each individual replicate was calculated as fold change with respect to the mean band intensity of liver samples (set as 1).

\subsection{Statistical analysis}

In Fig. 4 fold change values obtained for each CYP, time point, nicotine concentration and experiment are displayed as mean \pm standard deviation (SD). Different symbols are used to distinguish fold change values determined in different experiments with 3D aggregating brain cultures.

The responsiveness of the 3D cells to drug treatment was evaluated by calculating - separately for each CYP, cell batch and measurement time point - the Lowest Observed Effect Concentration (LOEC), i.e. the lowest concentration for which a fixed, pre-defined threshold for the fold change was exceeded. To derive this fixed threshold, all the fold change values that were obtained for CYP expression in untreated 3D cell cultures were log2-transformed. Then, arithmetic mean and standard deviation (SD) of all these $\log 2$ fold change values were calculated. Biological relevance limits for the $\log 2$ fold change values were set to mean $\pm 2 * \mathrm{SD}$. The two computed biological relevance limits were back-transformed to the original scale. Plots were created with GraphPad Prism. 
Table 1

Oligonucleotide primers and probes used for quantitative analysis of CYPs mRNA expression.

\begin{tabular}{|c|c|c|c|}
\hline Target gene & Primer/probe & Sequences $5^{\prime}$ to $3^{\prime}$ & Ref. \\
\hline \multirow[t]{3}{*}{ Cyclophilin A } & Forward & CTGATGGCGAGCCCTTG & This study \\
\hline & Reverse & TCTGCTGTCTTTGGAACTTTGTC & \\
\hline & Probe & CGCGTCTGCTTCGAGCTGTTTGCA & \\
\hline \multirow[t]{3}{*}{ CYP1A1 } & Forward & TGAGTTTGGGGAGGTTACTGGTT & Meredith et al. (2003) \\
\hline & Reverse & TGAAGGCATCCAGGGAAGAGT & \\
\hline & Probe & ATACCCAGCTGACTTCATTCCTATCCTCCGTT & \\
\hline \multirow[t]{3}{*}{ CYP1A2 } & Forward & TTTGGAGCTGGATTTGAAACAGT & \\
\hline & Reverse & TCATGAATCTTCCTCTGCACCTT & \\
\hline & Probe & ACAACAGCCATCTTCTGGAGCATTTTGCT & \\
\hline \multirow[t]{3}{*}{ CYP2B1 } & Forward & ACCGGCTACCAACCCTTGAT & \\
\hline & Reverse & TGTGTGGTACTCCAATAGGGACAA & \\
\hline & Probe & CCGCAGTAAAATGCCATACACTGATGCAG & \\
\hline \multirow[t]{3}{*}{ CYP3A1 } & Forward & CAGCAGCACACTTTCCTTTGTC & \\
\hline & Reverse & СTCCTCCTGCAGTTTCTTCTGTGTA & \\
\hline & Probe & TGCATTCCCTGGCCACTACC & \\
\hline \multirow[t]{3}{*}{ CYP3A2 } & Forward & GCTTTCAGCTCTCACACTGGAAA & \\
\hline & Reverse & TCTATGGGTTCCAAGTCGGTAGA & \\
\hline & Probe & TCCTCCTGGCAGTCATCCTGGTGC & \\
\hline \multirow[t]{2}{*}{ CYP2D2 } & Forward & TGAGTGGCGAGAGCAGAG & Mrozikiewicz et al. (2010) \\
\hline & Reverse & CGAGCATAAACAAGGGAGG & \\
\hline \multirow[t]{2}{*}{ CYP2E1 } & Forward & ССТТТСССТСТТСССАТСС & Mrozikiewicz et al. (2010) \\
\hline & Reverse & AACCTCCGCACATCCTTCC & \\
\hline \multirow[t]{2}{*}{ CYP2D4/2D6 } & Forward & AGCTTCAACACCGCTATGGT & Yoshida et al. (2002) \\
\hline & Reverse & CAGCAGTGTCCTCTCCATGA & \\
\hline
\end{tabular}

For the semi-quantitative determination of protein CYPs, the statistical evaluation of significance of fold changes with respect to the mean band intensity obtained for liver samples (set as 1) was performed using nonparametric Mann-Whitney $U$-test, and $P$-values below 0.5 were considered as significant.

\section{Results}

\subsection{Preliminary studies}

The appropriateness of three housekeeping genes (18S RNA, Cyclophilin A and Beta-actin) to be used as reference for the quantification of CYP levels was evaluated under different experimental conditions. Cyclophilin A was selected due to its stable expression characteristics. The concentrations of nicotine $(50-200 \mu \mathrm{M})$ were chosen in order to be <LOEC for toxicity determined after $44 \mathrm{~h}$ of exposure (Zurich et al., 2013).

\subsection{CYP expression in $3 D$ cell cultures}

CYPs mRNA expression levels were measured in untreated 3D cell cultures. Each CYP level was calculated as the mean value among the available different batches, and normalized with respect to the housekeeping Cyclophilin A content. In these control samples, CYP2B1, CYP1A1, CYP3A1, CYP2D2, CYP2D4/2D6 and CYP2E1 expression was clearly detectable, whereas the expression levels of CYP1A2 and CYP3A2 were below the limits of detection (data not shown). On average, relative mRNA content (with respect to the total CYP-mRNA) of CYP2B1, CYP1A1, CYP3A1, CYP2D2, CYP2D4/2D6 and CYP2E1 in untreated 3D cell cultures was highest for CYP1A1 (50\%) and CYP2B1 forms (27\%). Moderate expression levels were detected for CYP3A1 and CYP2D2 (10\% each), while only a small contribution from CYP2D4/2D6 (2\%) and CYP2E1 (1\%) was found (Fig. 1). Relative contents of CYP2E1, 2D4 and 2B1 were almost constant over three independent cell culture preparations, for both developmental stages (DIV20 and 33); CYP1A1 was highly variable, with levels between $30 \%$ and 54\% and CYP2D2 and $3 A 1$ varied from $8 \%$ to $15 \%$. Each value was reported as an average among culture replicates, among which some variability

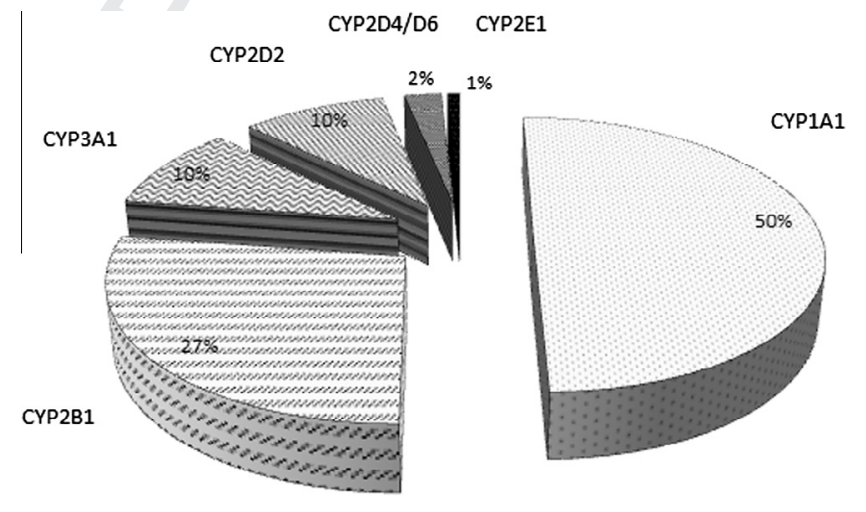

Fig. 1. Relative mRNA content of selected isoforms of CYP in untreated 3D aggregating brain cell cultures. The pie chart illustrates the relative proportion of expression (in \%) of CYP1A1, 2B1, 3A1, 2D2, 2D4/2D6 and 2E1 in control samples measured by qRT-PCR. The expression levels of CYP1A2 and CYP3A2 were below the limits of detection.

was shown (i.e. CV\% was $3-30 \%$ for CYP1A1 and $10-35 \%$ for CYP2B1).

\subsection{Spatial distribution and cell type-specific localization of CYPs}

Cellular localization of CYP1A1, 2B1/B2, 2D4, 2E1 and 3A was analyzed by immunohistochemistry using intracellular neurofilaments (NF) - phosphorylated and non-phosphorylated - and microtubule-associated protein-2 (MAP2) as neuronal markers, glial fibrillary acidic protein (GFAP) as astrocyte marker, and myelin basic protein (MBP) as a marker of oligodendrocytes.

CYP1A1 labeling mainly appeared around the cell nuclei and yellow co-localization signal was found with both NF and GFAP, and to some extent also with MBP (Fig. 2A), indicating CYP1A1 expression in neurons and astrocytes and to some extent also in oligodendrocytes.

CYP2D4 labeling had primarily a cell-body and cellular process distribution (Fig. 2B). Some perinuclear and somal CYP2D4 labeling co-localized with NF, whereas somal and cell process CYP2D4 
highly co-localized with GFAP (Fig. 2B, insets), indicating that CYP2D4 is primarily expressed in astrocytes. Only faint CYP2D4 co-localization was seen with MBP (Fig. 2B).

Labeling of CYP2E1 was also located around the nucleus and in the cell body: co-localization with NF was primarily perinuclear as shown by overlapping Hoechst labeling and CYP2E1-NF co-localization (Fig. 2C, inset). CYP2E1-GFAP co-localization appeared primarily in the cell body (Fig. 2C, insets). CYP2E1 co-localization signal with MBP was weak (Fig. 2C), suggesting primarily neuronal and astroglial localization of CYP2E1.
380
A

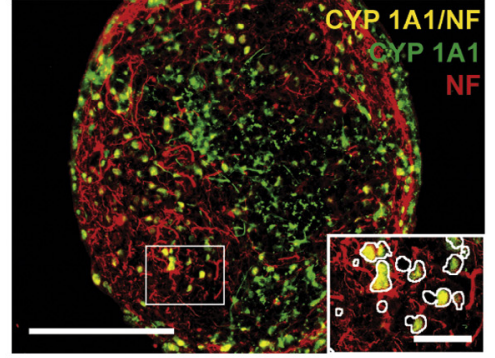

B

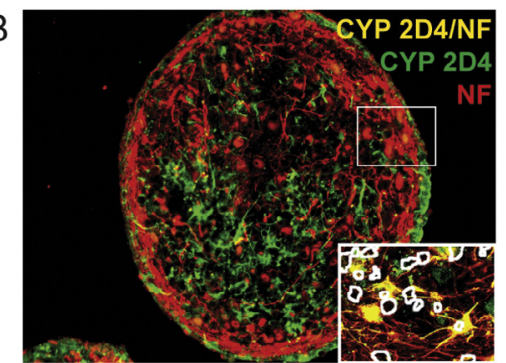

C

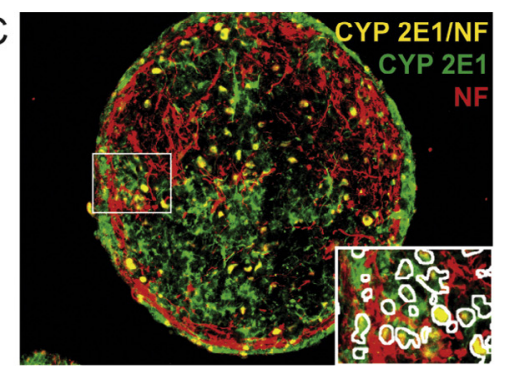

D

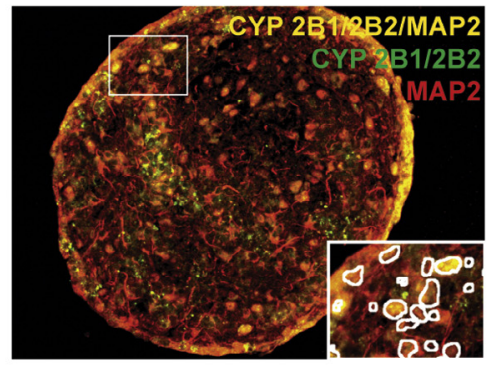

E

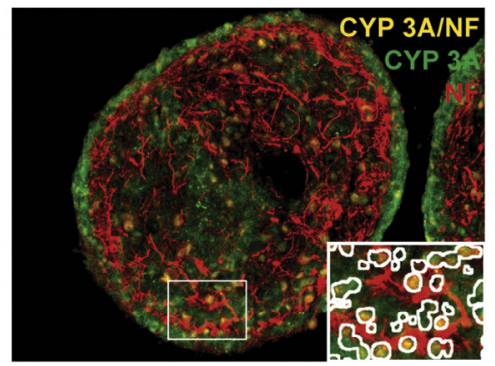

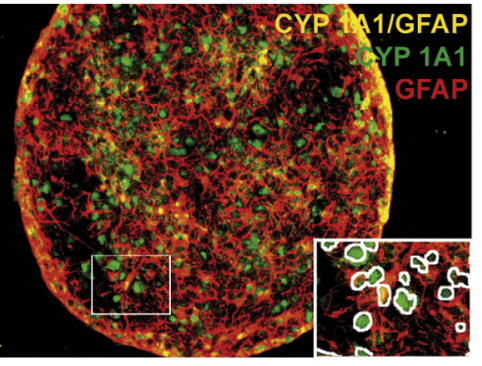
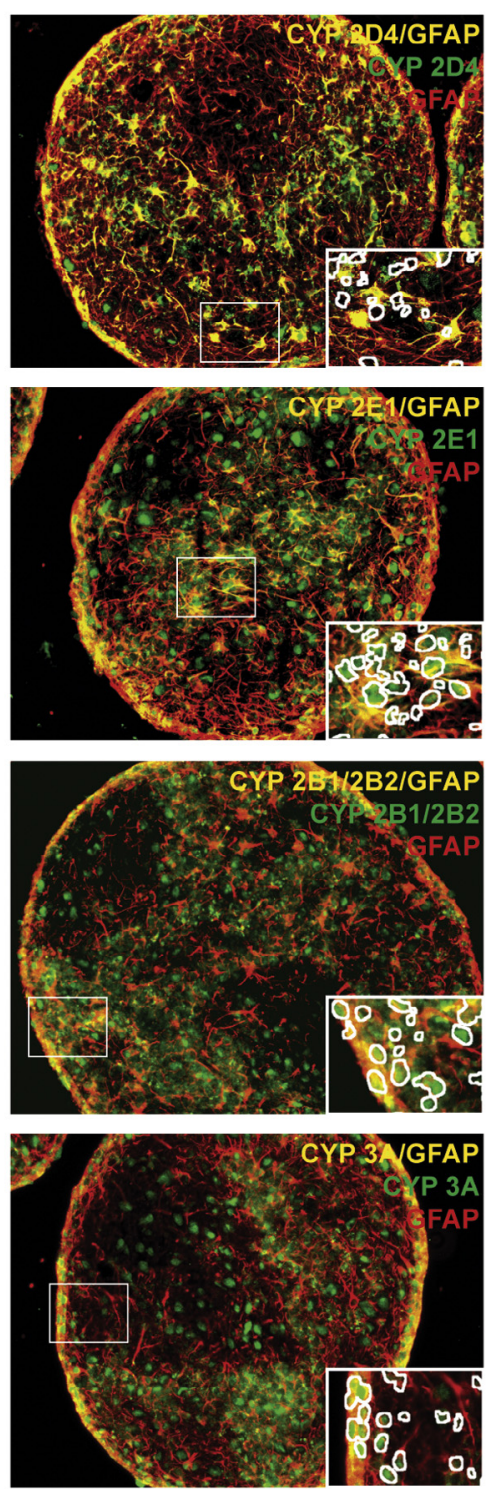
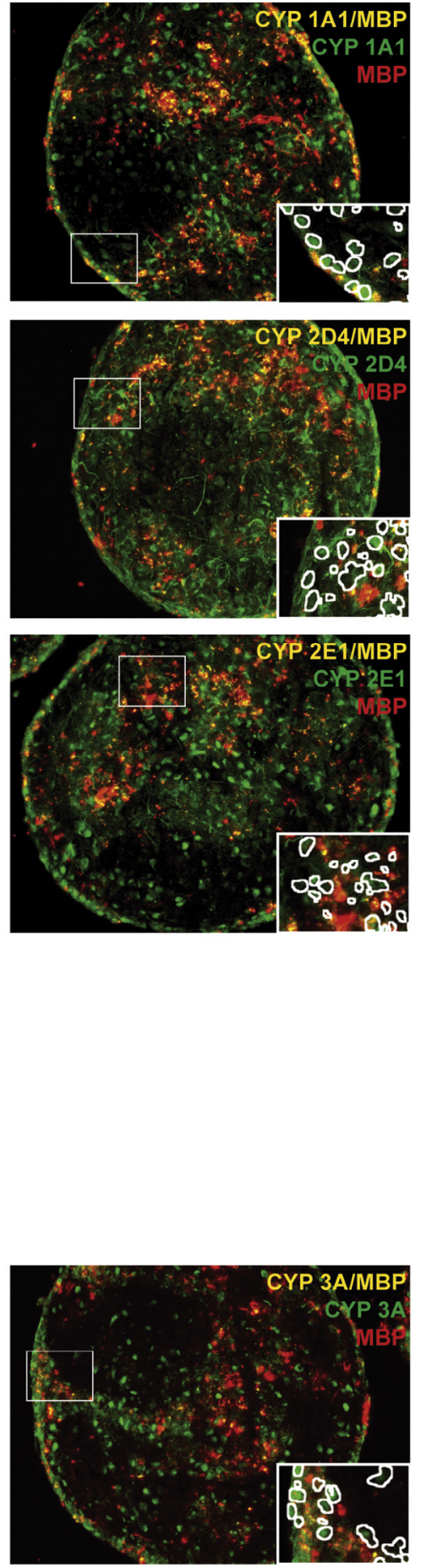

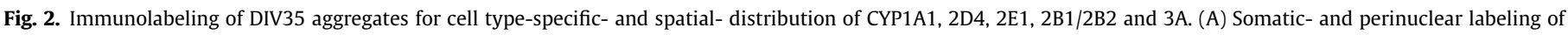

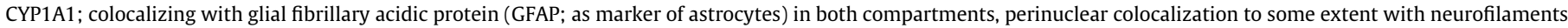

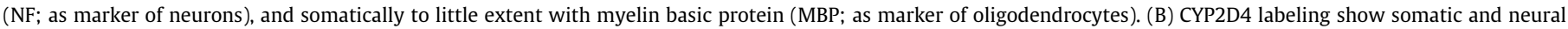

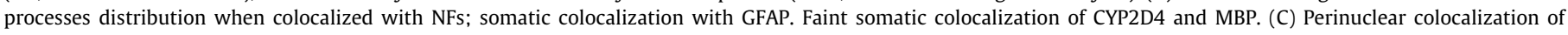

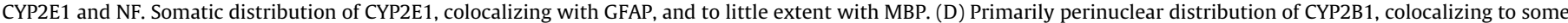

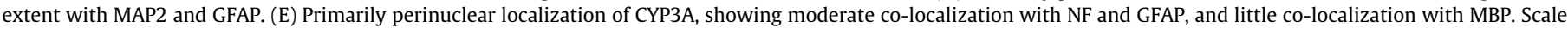
bar indicates $100 \mu \mathrm{m}$, and $20 \mu \mathrm{m}$ in the inset. White lines encircle the nuclear localization revealed by Hoechst.

Please cite this article in press as: Vichi, S., et al. Cell type-specific expression and localization of cytochrome P450 isoforms in tridimensional aggregating rat brain cell cultures. Toxicol. in Vitro (2015), http://dx.doi.org/10.1016/j.tiv.2015.03.005 
Labeling signal of CYP2B1/2B2 was low and primarily distributed around the nuclei, as indicated by overlapping with Hoechst; co-localization could be detected to some extent with both MAP2 and GFAP (Fig. 2D, insets), indicating CYP2B1/2B2 expression in both neurons and astrocytes (not tested in oligodendrocytes).

Finally, CYP3A labeling was very weak and primarily distributed around the nuclei (Fig. 2E and inset). Little CYP3A co-localization was found with NF and MBP, whereas GFAP co-localized to a greater extent (Fig. 2E, insets), indicating that CYP3A expression is mainly astrocytic.

\subsection{Relative CYP-protein content in aggregating brain cell cultures as} compared to adult liver and brain

Relative protein levels of CYP1A1, 2D4, 2E1, 2B1/2B2 and 3A1 were analyzed in whole protein lysates of adult rat liver, adult rat brain and in vitro mature aggregating brain cell cultures. The quantification of band intensity for each CYP-isoform is expressed relatively to the band intensity found in adult liver homogenates (Fig. 3).

CYP1A1 antibody revealed a band, corresponding to its predicted molecular weight of $55 \mathrm{kDa}$ (Fig. 3A, lower panel) and band intensities in brain and aggregate cultures appeared stronger as compared to the liver (approximately 5- to 6-fold, Fig. 3B). The same trend was observed in the case of CYP2D4, where - although highly variable - the corresponding $56 \mathrm{kDa}$-band was more intense in brain and aggregate culture lysates as compared to liver (Fig. 3A lower panel, and B). However, despite this variability, CYP1A1 and 2D4 detection in aggregates seem to follow the same tendency as the in vivo brain, with higher quantities as compared to liver.

CYP2E1, 2B1/2B2 and 3A1 bands were detected at their respective molecular sizes of $60-, 50$ - and $50 \mathrm{kDa}$ in liver-, brain- and aggregate culture lysates (Fig. 3A, lower panel). However, when quantified, band intensities for all three proteins were weaker in brain and aggregate culture lysates as compared to liver (Fig. 3B). Overall, this indicates that the three CYP-isoforms are weakly expressed in the brain cells as compared to liver. Again, CYP2E1, 2B1/B2 and 3A1 expression in aggregates appears comparable to that of in vivo brain.

\subsection{Inducibility of CYPs by nicotine treatment}

The responsiveness of the 3D cell cultures to nicotine treatment was evaluated as outlined in the statistical analysis section. As outcome of these computations, the following biological relevance limits were obtained: lower limit $=0.677$-fold, upper limit $=1.429$-fold. These two limits are indicated by the horizontal lines that are displayed in each of the six panels of Fig. 4.

For CYP2B1, in five experiments (II-VI) with 3D cell cultures prepared from three independent pools of embryonic rat brains, no up-regulation of CYP2B1 expression was observed after nicotine treatment (Fig. 4, upper panel). Only in experiment I, performed on aggregates coming from a fourth pool of embryonic brains, a consistent induction of CYP2B1 was observed upon 24 and 48 h nicotine treatment (Fig. 4). Here, 48 h-treatment increased CYP2B1 expression up to 13.5 -fold, as compared to the constitutive CYP2B1. In summary, excluding results from experiment I, statistical evaluation of CYP2B1 expression data failed to show a consistent and biologically relevant induction effect after nicotine treatment.

Statistical evaluation of CYP1A1 expression indicate minute induction capability of nicotine, with fold change values above the upper threshold for at least one nicotine concentration and time point in three out of six experiments (Fig. 4, lower panel). Again, limited to batch I, a biologically relevant induction after 24 - and 48 h nicotine treatment was also observed (Fig. 4), with up to 2.5-fold increased mRNA expression as compared to controls. However, this increase was not concentration dependent and showed only a modest trend toward higher levels with increasing time.

CYP1A1 and 2B1 expression levels observed in control samples and after nicotine treatment were comparable between the tested developmental stages (DIV 20 and DIV 33) (Fig. 4).
A
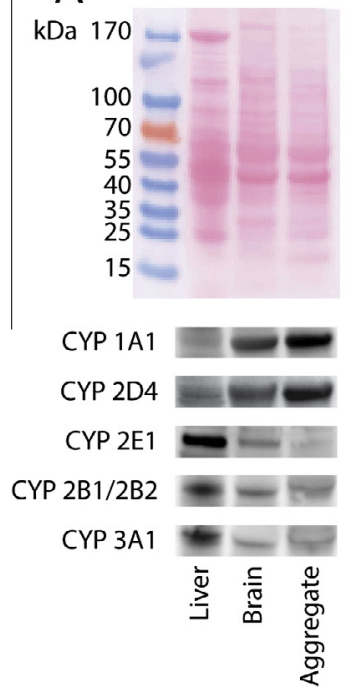

B

CYP 1A1 CYP 2D4

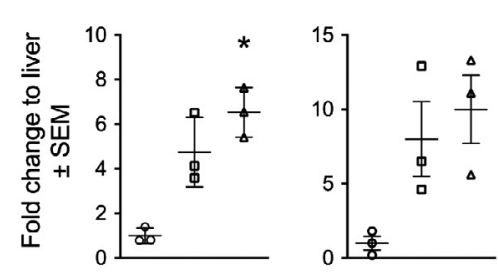

CYP 2E1

CYP 2B1/2B2

CYP $3 A 1$

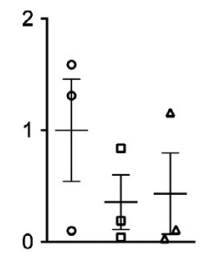

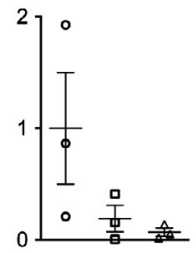

- Liver

- Brain

$\triangle$ Aggregate

* $P<0.5$

** $P<0.05$

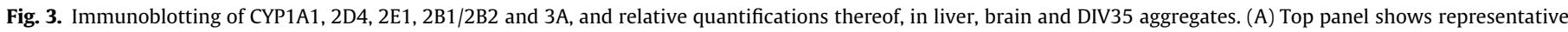

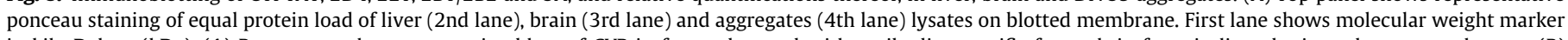

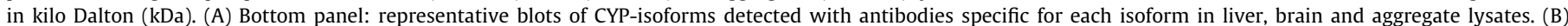

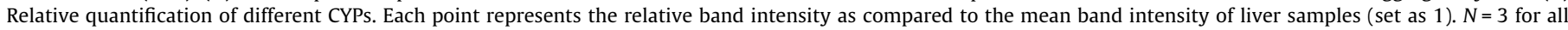
tissues. The statistical significance of fold changes was performed using nonparametric Mann-Whitney $U$-test; $P$-values below 0.5 were considered as significant. 

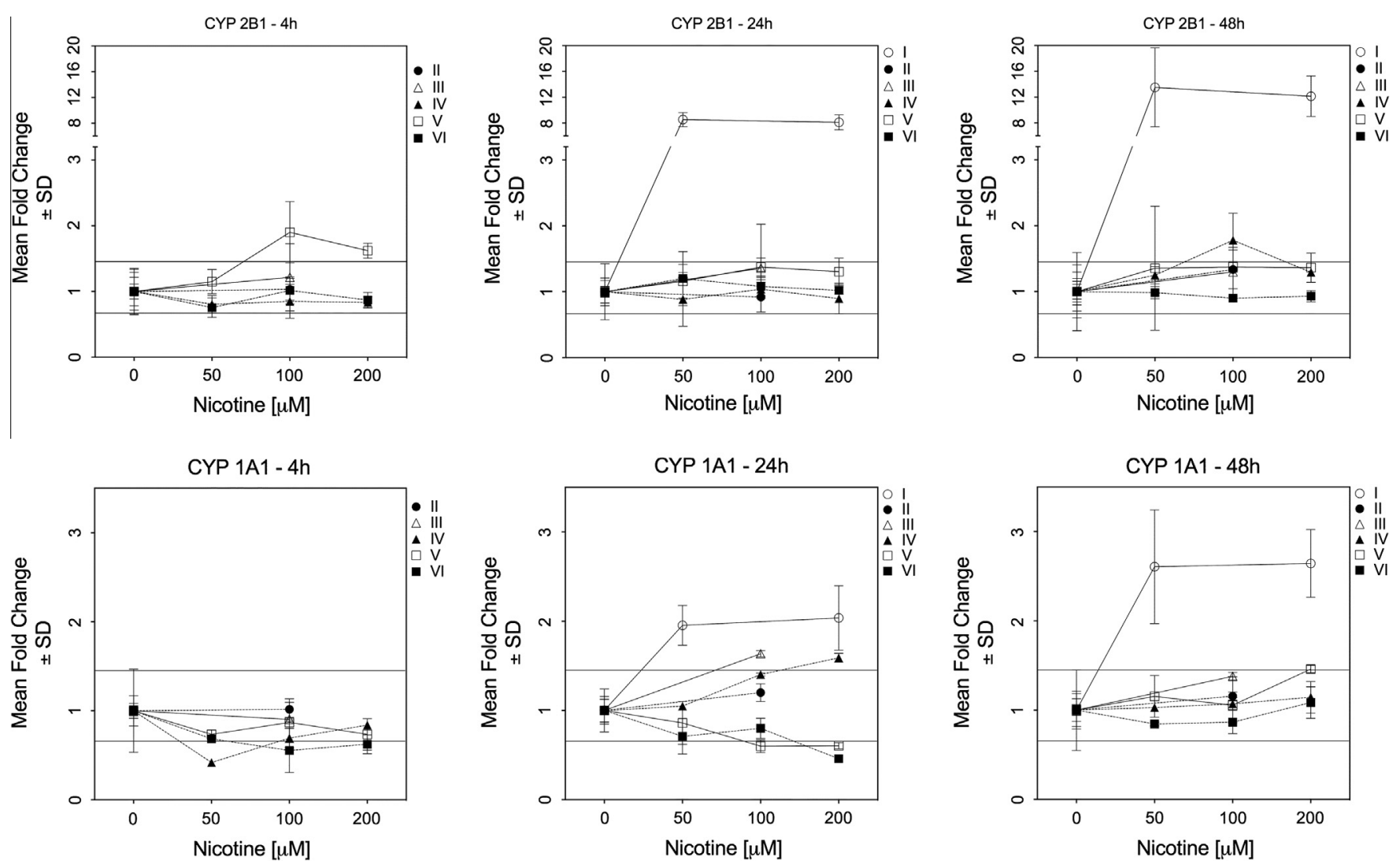

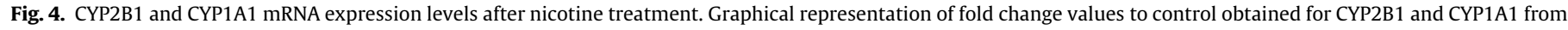

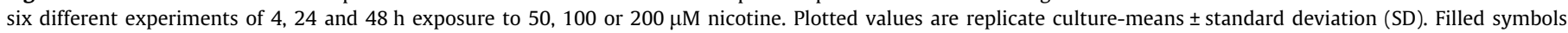
connected with dotted lines indicate DIV20, and open symbols connected with full lines DIV33.

\section{Discussion}

To our knowledge, this is the first study aimed to characterize CYP-content of 3D brain cell cultures. The constitutive metabolic competence is a particularly important feature, knowing that the drug metabolizing CYP-dependent system in the brain can play a crucial role in the local biotransformation of endogenous and exogenous compounds, such as centrally acting drugs, neurotoxins, and neurochemicals.

The two major limitations in using results from in vitro models to evaluate the risk related to neurotoxic effects of chemicals, or safety during drug development, is the complexity of the cell types characterizing the brain, in addition to scant knowledge of the biokinetic parameters. Neurotoxicity has been identified as a frequent adverse drug effect, contributing to the termination of up $22 \%$ of drug candidates (Watkins, 2011). It is known that bioavailability/biokinetics of a chemical is a key element in eliciting toxic effects for a drug candidate. Indeed, most CNS-targeting drugs have been withdrawn because of poor blood brain barrier permeability; once demonstrated they reach the target, the insufficient characterization of metabolic competence of the available model does not allow an estimation of the possible in situ biotransformation.

Aggregating brain cell cultures prepared from embryonic rat brains address the first issue, containing different types of brain cells (i.e., neurons, astrocytes, oligodendrocytes, and microglia), able to grow and mature in serum-free, chemically defined medium, and form highly differentiated structures and functional neuronal networks (Honegger et al., 1979). The histotypic organization of the cells within the 3D structure is close to the situation in vivo; thus, 3D aggregating brain cell cultures constitute a useful system for neurotoxicological studies. The model allows detection of toxicity of chemicals affecting selectively either the neurons or the glial cells after acute and repeated exposure (Zurich et al., 2013; Bellwon et al., in press; Pomponio et al., in press). Furthermore, organophosphorous pesticides such as parathion and chlorpyrifos, requiring metabolic activation via cytochrome $\mathrm{P} 450$, exhibited organ-specific toxicity (Monnet-Tschudi et al., 2000), indirectly suggesting that cells in the model possess some metabolic activity.

The present results clearly indicate that several CYPs, namely 1A1, 2B1, 3A, 2D2, 2D4 and 2E1, are constitutively expressed in $3 \mathrm{D}$ aggregating brain cell cultures, with the first two in the list accounting for $2 / 3$ of the total CYP-mRNA detected. The establishment of relative proportions of the different isoforms cannot be made at the protein levels in this study, since they were not detected as absolute content, but only quantified relatively to liver homogenates. Moreover, in analogy to the in vivo situation (Miksys et al., 2000; Miksys and Tyndale, 2002; Ravindranath et al., 1995), CYP proteins are differently localized in the various cell types.

Regarding CYP1A1, our data, showing localization in both neurons and astrocytes and scarcely in oligodendrocytes, are in agreement with published data indicating a significant mRNA and protein expression of CYP1A1 in cultured rat brain neurons and glial cells (Kapoor et al., 2006). As shown by western blot, CYP1A1 content in brain as well as in 3D aggregates is higher than in liver, where constitutive expression is described to be low (Drahushuk et al., 1998; Chinta et al., 2005), this isoform being mainly an extra-hepatic isoform. The presence of CYP2B1 has been shown in different regions of rat brain, in neurons and glial cells (Miksys et al., 2000; Rosenbrock et al., 2001; Volk et al., 1991). Also in this case our results show that the in vitro model maintains similar features for CYP2B expression, with localization in both 
glial and neuronal cell populations, likely distributed in the perinuclear area. CYP2B protein detection was highly variable in liver-, brain- and aggregate samples, not allowing to draw definite conclusions on the relative content. For CYP2E1 and CYP3A, moderate to low levels of mRNA were measured in 3D cultures, with barely detectable protein amounts of respective CYPs compared to liver and a marked difference in cellular distribution. In vivo, CYP2E1 was found in the midbrain in cells with the morphological characteristics of dopaminergic neurons (Riedl et al., 1996), while various results were reported regarding its presence in astrocytes (Hansson et al., 1990; Watts et al., 1998). In aggregating rat brain cell cultures, CYP2E1 was localized in both neurons and astrocytes. For CYP3A, results are in line with in vivo data showing a lower expression of CYP3A in brain than in liver (Woodland et al., 2008), although inter-individual variability of CYP3A expression in liver appears to be high. In the brain aggregates, CYP3A was found mainly in astrocytes whereas limited information is available on its in vivo cellular localization. For CYP2D4, protein expression appeared concentrated mainly in astrocytes, while less was found in neurons and oligodendrocytes. In this study - as previously reported in vivo (Hiroi et al., 1998; Chinta et al., 2002) CYP2D4 levels were higher in brain tissue as compared to liver, again CYP2D4 levels in 3D brain cell cultures were similar to brain.

In vivo, chronic exposure to nicotine specifically induces brain CYPs, mainly in neurons (Jacob et al., 1997; Miksys et al., 2000; Zevin and Benowitz, 1999), whereas the total CYP content in the liver remains unaffected (Anandatheerthavarada et al., 1993; Miksys et al., 2000; Yue et al., 2008). Neurons and glial cells in vitro have also been shown to respond to CYP1A1/1B1 inducers (Jacob et al., 1997; Kapoor et al., 2006; Miksys et al., 2000).

In the present study, the quantification of CYP1A1 and CYP2B1 mRNA levels was used as a marker of induction, as previously reported (Meredith et al., 2003; Pavek and Dvorak, 2008). However, after short-term exposure to nicotine mRNA content showed highly variable results. A plausible explanation arises from the observed variations in the relative proportions of the constitutive CYPs among culture preparations, possibly due to variations in the proportions of the different type of cells, or to individual variations in the rat embryos used to prepare the cultures, as well as to the short duration of exposure to nicotine. Indeed, strong induction of CYP2B1 has been reported after longterm treatment with nicotine (Khokhar et al., 2010). Our data shows the potentiality of 3D aggregating brain cells to be responsive to inducers, although before concluding on this issue, longer exposure time and other prototype inducers should be used. Furthermore, protein levels after exposure to inducers should be monitored. In this respect, preliminary results showed increased immunolabeling of CYP1A1 after $48 \mathrm{~h}$ exposure to nicotine (not shown).

In the present work, no evidence of mRNA induction after nicotine treatment was found for CYP2E1, CYP3A AND CYP2D families. Consistently, after nicotine exposure the induction of brain CYP2E1 and $2 \mathrm{D}$ has been reported in absence of mRNA increase, suggesting nontranscriptional regulation (Joshi and Tyndale, 2006; Yue et al., 2008).

The present findings, showing the expression of CYP1A1, CYP2B1/B2, CYP2D4, CYP2E1 and CYP3A isoforms in 3D aggregating rat brain cell cultures, together with previously reported observations on the deleterious effects of organophosphorous pesticides (Monnet-Tschudi et al., 2000; Zurich et al., 2000) not simply attributable to the parent compounds, suggest that this model possesses metabolic capacity. In addition, the recently reported formation of the major oxidative metabolite of amiodarone in 3D aggregating rat brain cell cultures after 14-day repeated exposure (Pomponio et al., in press) constitutes a direct demonstration of the functionality of the detected CYPs. Indeed, the isoforms known to metabolise amiodarone, an antiarrhythmic drug causing neurotoxic side-effects in some patients following long-term treatment, belong to the CYP3A, 2C, 1A and 2D family in rodents and in humans (Zahno et al., 2011), and most of them have been demonstrated here to be constitutively expressed in this 3D cell culture model.

Although requiring further confirmation by measuring CYP-activity, these results suggest an intrinsic metabolic capacity, most likely contributing to the high performance of this culture model in neurotoxicological studies, and support its use in studying brain toxication/detoxication mechanisms.

\section{Conflict of Interest}

The authors declare that there are no conflicts of interest.

\section{Transparency Document}

The Transparency document associated with this article can be found in the online version.

\section{Uncited reference}

Lowry et al. (1951).

\section{Acknowledgments}

This project was funded by the European Union's 7th Framework Programme (FP7/2007-2013) under grant agreement no 202222, Predict-IV. The administrative and organization skills of Hannelore Popa-Henning are also much appreciated.

\section{References}

Anandatheerthavarada, H.K., Williams, J.F., Wecker, L., 1993. The chronic administration of nicotine induces cytochrome $\mathrm{P} 450$ in rat brain. J. Neurochem. 60, 1941-1944.

Ande, A., Earla, R., Jin, M., Silverstein, P.S., Mitra, A.K., Kumar, A., Kumar, S., 2012. An LC-MS/MS method for concurrent determination of nicotine metabolites and the role of CYP2A6 in nicotine metabolite-mediated oxidative stress in SVGA astrocytes. Drug Alcohol Depend. 125, 49-59.

Bellwon, P., Culot, M., Wilmes, A., Schmidt, T., Zurich, M.-G., Schultz, L., Schmal, O. Gramowski-Voss, A., Weiss, D.G., Jennings, P., Bal-Price, A., Testai, E., Dekant, W. in press. Cyclosporine A kinetics in brain cell cultures and its potential of crossing the blood-brain barrier. Toxicol. In Vitro (2015).

Chinta, S.J., Pai, H.V., Upadhya, S.C., Boyd, M.R., Ravindranath, V., 2002. Constitutive expression and localization of the major drug metabolizing enzyme cytochrome P4502D in human brain. Mol. Brain Res. 103, 49-61.

Chinta, S.J., Kommaddi, R.P., Turman, C.M., Strobel, H.W., Ravindranath, V., 2005 Constitutive expression and localization of cytochrome P-450 1A1 in rat and human brain: presence of a splice variant form in human brain. J. Neurochem. 93, 724-736.

Drahushuk, A.T., McGarrigle, B.P., Larsen, K.E., Stegeman, J.J., Olson, J.R., 1998 Detection of CYP1A1 protein in human liver and induction by TCDD in precision-cut liver slices incubated in dynamic organ culture. Carcinogenesis 19, 1361-1368.

Fletcher, A.P., 1978. Drug safety tests and subsequent clinical experience. J. R. Soc Med. 71, 693-696.

Ghersi-Egea, J.F., Walther, B., Perrin, R., Minn, A., Siest, G., 1987. Inducibility of rat brain drug-metabolizing enzymes. Eur. J. Drug Metab. Pharmacokinet. 12, 263265.

Hansson, T., Tindberg, N., Ingelman-Sundberg, M., Kohler, C., 1990. Regional distribution of ethanol-inducible cytochrome P450 IIE1 in the rat centra nervous system. Neuroscience 34, 451-463.

Harry, G.J., Tiffany-Castiglioni, E., 2005. Evaluation of neurotoxic potential by use of in vitro systems. Expert Opin. Drug Metab. Toxicol. 1, 701-713.

Hiroi, T., Imaoka, S., Chow, T., Funae, Y., 1998. Tissue distributions of CYP2D1, 2D2, 2D3 and 2D4 mRNA in rats detected by RT-PCR. Biochim. Biophys. Acta 1380, 305-312.

Honegger, P., Werffeli, P., 1988. Use of aggregating cell cultures for toxicological studies. Experientia 44, 817-823.

Honegger, P., Lenoir, D., Favrod, P., 1979. Growth and differentiation of aggregating fetal brain cells in a serum-free defined medium. Nature $282,305-308$.

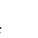

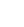

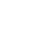

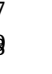

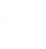

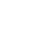

1

2

3


Honegger, P., Defaux, A., Monnet-Tschudi, F., Zurich, M.G., 2011. Preparation, maintenance, and use of serum-free aggregating brain cell cultures. Methods Mol. Biol. 758, 81-97.

Jacob 3rd, P., Ulgen, M., Gorrod, J.W., 1997. Metabolism of (-)-(S)-nicotine by guinea pig and rat brain: identification of cotinine. Eur. J. Drug Metab. Pharmacokinet. 22, 391-394.

Johri, A., Yadav, S., Dhawan, A., Parmar, D., 2007. Overexpression of cerebral and hepatic cytochrome P450s alters behavioral activity of rat offspring following prenatal exposure to lindane. Toxicol. Appl. Pharmacol. 225, 278-292.

Joshi, M., Tyndale, R.F., 2006. Induction and recovery time course of rat brain CYP2E1 after nicotine treatment. Drug Metab. Dispos. 34, 647-652.

Kapoor, N., Pant, A.B., Dhawan, A., Dwievedi, U.N., Seth, P.K., Parmar, D., 2006 Cytochrome P450 1A isoenzymes in brain cells: expression and inducibility in cultured rat brain neuronal and glial cells. Life Sci. 79, 2387-2394.

Khokhar, J.Y., Miksys, S.L., Tyndale, R.F., 2010. Rat brain CYP2B induction by nicotine is persistent and does not involve nicotinic acetylcholine receptors. Brain Res. 1348, 1-9.

Lowry, O.H., Rosebrough, N.J., Farr, A.L., Randall, R.J., 1951. Protein measurement with the Folin phenol reagent. J. Biol. Chem., 265-275

Meredith, C., Scott, M.P., Renwick, A.B., Price, R.J., Lake, B.G., 2003. Studies on the induction of rat hepatic CYP1A, CYP2B, CYP3A and CYP4A subfamily form mRNAs in vivo and in vitro using precision-cut rat liver slices. Xenobiotica 33, 511-527.

Meyer, R.P., Gehlhaus, M., Knoth, R., Volk, B., 2007. Expression and function of cytochrome p450 in brain drug metabolism. Curr. Drug Metab. 8, 297306.

Miksys, S.L., Tyndale, R.F., 2002. Drug-metabolizing cytochrome P450s in the brain. J. Psychiatry Neurosci. 27, 406-415.

Miksys, S.L., Tyndale, R.F., 2004. The unique regulation of brain cytochrome P450 2 (CYP2) family enzymes by drugs and genetics. Drug Metab. Rev. 36, 313333.

Miksys, S., Hoffmann, E., Tyndale, R.F., 2000. Regional and cellular induction of nicotine-metabolizing CYP2B1 in rat brain by chronic nicotine treatment Biochem. Pharmacol. 59, 1501-1511.

Monnet-Tschudi, F., Zurich, M.G., Schilter, B., Costa, L.G., Honegger, P., 2000 Maturation-dependent effects of chlorpyrifos and parathion and their oxygen analogs on acetylcholinesterase and neuronal and glial markers in aggregating brain cell cultures. Toxicol. Appl. Pharmacol. 165, 175-183.

Monnet-Tschudi, F., Hazekamp, A., Perret, N., Zurich, M.G., Mangin, P., Giroud, C., Honegger, P., 2008. Delta-9-tetrahydrocannabinol accumulation, metabolism and cell-type-specific adverse effects in aggregating brain cell cultures. Toxicol Appl. Pharmacol. 228, 8-16.

Parmar, D., Yadav, S., Dayal, M., Johri, A., Dhawan, A., Seth, P.K., 2003. Effect of lindane on hepatic and brain cytochrome P450s and influence of P450 modulation in lindane induced neurotoxicity. Food Chem. Toxicol. 41, 10771087.

Pavek, P. Dvorak, Z., 2008. Xenobiotic-induced transcriptional regulation of xenobiotic metabolizing enzymes of the cytochrome P450 superfamily in human extrahepatic tissues. Curr. Drug Metab. 9, 129-143.
Pomponio, G., Zurich, M.-G., Schultz, L., Weiss, D.G., Romanelli, L., Gramowski-Voss, A. Di Consiglio, E., Testai, E. in press. Amiodarone biokinetics, the formation of its major oxidative metabolite and neurotoxicity after acute and repeated exposure of brain cell cultures. Toxicol. In Vitro (2015).

Ravindranath, V., Strobel, H.W., 2013. Cytochrome P450-mediated metabolism in brain: functional roles and their implications. Expert Opin. Drug Metab. Toxicol. 9, 551-558.

Ravindranath, V., Bhamre, S., Bhagwat, S.V., Anandatheerthavarada, H.K., Shankar, S.K., Tirumalai, P.S., 1995. Xenobiotic metabolism in brain. Toxicol. Lett. 82-83, 633-638.

Rieder, C.R., Ramsden, D.B., Williams, A.C., 1998. Cytochrome P450 1B1 mRNA in the human central nervous system. Mol. Pathol. MP51, 138-142.

Riedl, A.G., Watts, P.M., Edwards, R.J., Boobis, A.R., Jenner, P., Marsden, C.D., 1996. Selective localisation of P450 enzymes and NADPH-P450 oxidoreductase in rat basal ganglia using anti-peptide antisera. Brain Res. 743, 324-328.

Rosenbrock, H., Hagemeyer, C.E., Ditter, M., Knoth, R., Volk, B., 2001. Expression and localization of the CYP2B subfamily predominantly in neurones of rat brain. J. Neurochem. 76, 332-340.

Stamou, M., Wu, X., Kania-Korwel, I., Lehmler, H.-J., Leim, P.J., 2014. Cytochrome P450 mRNA expression in the rodent brain: species-, sex-, and regiondependent differences. Drug Metab. Dispos. 42, 239-244.

Volk, B., Hettmannsperger, U., Papp, T., Amelizad, Z., Oesch, F., Knoth, R., 1991. Mapping of phenytoin-inducible cytochrome P450 immunoreactivity in the mouse central nervous system. Neuroscience 42, 215-235.

Watkins, P.B., 2011. Drug safety sciences and the bottleneck in drug development. Clin. Pharmacol. Ther. 89, 788-790.

Watts, P.M., Riedl, A.G., Douek, D.C., Edwards, R.J., Boobis, A.R., Jenner, P., Marsden, C.D., 1998. Co-localization of P450 enzymes in the rat substantia nigra with tyrosine hydroxylase. Neuroscience $86,511-519$.

Woodland, C., Huang, T.T., Gryz, E., Bendayan, R., Fawcett, J.P., 2008. Expression, activity and regulation of CYP3A in human and rodent brain. Drug Metab. Rev. 40, 149-168.

Yue, J., Miksys, S., Hoffmann, E. Tyndale, R.F., 2008. Chronic nicotine treatment induces rat CYP2D in the brain but not in the liver: an investigation of induction and time course. J. Psychiatry Neurosci. JPN 33, 54-63.

Zanger, U.M., Raimundo, S., Eichelbaum, M., 2004. Cytochrome P450 2D6: overview and update on pharmacology, genetics, biochemistry. Naunyn-Schmiedeberg's Arch. Pharmacol. 369, 23-37.

Zevin, S., Benowitz, N.L., 1999. Drug interactions with tobacco smoking. An update. Clin. Pharmacokinet. 36, 425-438.

Zurich, M.G., Monnet-Tschudi, F., Berode, M., Honegger, P., 1998. Lead acetate toxicity in vitro: dependence on the cell composition of the cultures. Toxicol. In Vitro 12, 191-196.

Zurich, M.G., Honegger, P., Schilter, B., Costa, L.G., Monnet-Tschudi, F., 2000. Use of aggregating brain cell cultures to study developmental effects of organophosphorus insecticides. Neurotoxicology 21, 599-605.

Zurich, M.G., Stanzel, S., Kopp-Schneider, A., Prieto, P., Honegger, P., 2013. Evaluation of aggregating brain cell cultures for the detection of acute organspecific toxicity. Toxicol. In Vitro 27, 1416-1424. 